\title{
Influence of unbalanced electromagnetic force and air supply pressure fluctuation in air bearing spindles on machining surface topography
}

\author{
Yazhou Sun ${ }^{1}$, Quanhui Wu',2\#, Wanqun Chen 1 , Xichun Luo ${ }^{3}$ and Guoda Chen² \\ 1 School of Mechatronics Engineering, Harbin Institute of Technology, Harbin 150001, P. R. China \\ 2 Key Laboratory of E\&M, Ministry of Education \& Zhejiang Province, Zhejiang University of Technology, Hangzhou 310032, P. R. China \\ 3 Centre for Precision Manufacturing, DMEM, University of Strathclyde, Glasgow G1 1XJ, UK \\ \# Corresponding Author / E-mail: wuqh@zjut.edu.cn/sunyzh@hit.edu.cn, TEL: + 86-0451-86413840, FAX: + 86-0451-86415244
}

KEYWORDS : Air Bearing Spindles, Radial magnetic force, Air pressure variation, Surface topography

\begin{abstract}
Air Bearing Spindles (ABS) can meet high accuracy demands for the precise rotation motion, which adopt air bearings to support spindle shaft, and the spindle shaft directly connects the motor rotor. The unbalanced electromagnetic force caused by motor rotor eccentricity (MRE) and air pressure fluctuation (APF) are two important influential factors to the dynamic performance of the spindle system and machining surface quality. This paper addresses the problems of measuring the MRE and APF in an ABS through testing machining surface topography. A permanent magnet synchronous motor (PMSM) was modelled by finite element simulation (FES). Through FES it found that the MRE between the motor rotor and stator hole produced a Radial Magnetic Force (RMF), which could cause ABS to periodically vibrate in the axial direction. Besides, the change of the air supply caused the stiffness variation of ABS and result in the tilt error motions of the spindle shaft. A theoretical model of machining surface topography considering MRE and APF was then proposed for the first time, which revealed that the MRE and APF resulted in the periodic fluctuations of the machining surface topography. The overall surface topography then became grooved surfaces. The above findings were finally validated by measurement results of ultraprecision diamond turning experiments.
\end{abstract}

Manuscript received: August XX, 201X / Accepted: August XX, 201X

\section{Introduction}

Air Bearing Spindles (ABS) plays a decisive role in the machining performance of ultra-precision machine tools. The schematic diagram of an ABS is shown in Fig. 1, a permanent magnet synchronous motor (PMSM) is set as the drive source, and air bearings are set as the supporting structure. Because ABS has the special structure, the manufacturing imperfections and assembly misalignments are prone to affect the rotation accuracy of the spindle system, and introduce easily unwanted offset motions such as radial, axial, or tilt motions. In addition, because ABS adopts air bearings as the spindle, air supply plays an important role in ABS, and the value change of air pressure affects the bearing stiffness, which also affects the machining productivity and quality of the workpiece surface. Many scholars have studied the imbalanced air bearings performance[1-3], however, there are few studies on the influence of motor and air pressure problems on machining.

In ideal condition of PMSM, the stator and motor rotor axes are coincident, and the electromagnetic force between stator and rotor acting upon the rotor surface is a balanced state [4]. Nevertheless, because of the tolerance in manufacture and assembly, motor rotor eccentricity (MRE) between stator and motor rotor is unavoidable

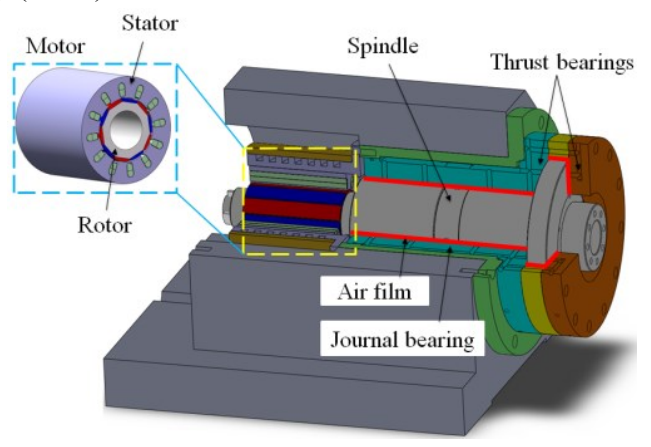

Fig.1 Schematic view of an ABS

in electric machines[5,6]. If the axis position of the motor rotor is 
displaced from the center of the stator bore, the air-gap magnetic field becomes unevenly distributed, and there will be a Radial Magnetic Force (RMF) acting on the motor rotor. RMF is usually considered as bad phenomenon, because it could degrade the motor performance, which could cause vibrations and acoustic noise to ABS. Recently, many researchers have reported the electromagnetic vibrations in motors[7,8]. To reduce the vibrations and noise, the excitation frequencies of the torque ripple and RMF induced by various sources are identified[9], and effects of MRE and vibration modes on fractional slot surface-mounted PM brushless machines are systematically investigated, which had different slot-pole numbers[10]. To reduce the vibrations caused by MRE, a method of introducing parallel paths in windings is proposed to reduces RMF and vibrations[11]. Admittedly, some experts have analyzed different motor characteristics, RMF has a significant effect on the machining quality, but few studies have been carried out on motors impacts of ABS and the actual machining. Previous work has done some research on MRE of a vertical ultra-precision spindle, a ABS for potassium dihydrogen phosphate crystal machining tool was selected as the research object, and a range of MRE was calculated to assess the impact of the radial magnetic force on the spindle performance[12]. It is found that MRE has a significant influence on the spindle vibration, which dramatically reduces the processing quality. The horizontal spindles and vertical spindles have different characteristics, such as centroid offset is a common phenomenon for horizontal spindles. In this paper, combining the analysis theory of the vertical ultraprecision spindle, the characteristics of the horizontal spindle are studied considering MRE and air supply pressure.

At present, ultra-precision spindles have been studied for many dynamic characteristics, both theoretically and experimentally. A dynamic spindle model was established to describe its dynamic responses, involving translational motions and tilting motions[13], and the structure of an ultra-precision spindle for crystal machining was designed and optimized[14,15]. To deeply understand the spindle performance and improve the machining quality, the measurement and analysis of the ultra-precision spindle were useful methods[16,17]. A lot of research have been done on the dynamic characteristics of the spindle system[18], but some key technical problems are still not solved. For example, there are inadequate for identifying the contribution of spindle drift, especially how MRE impacts on ABS in terms of machining accuracy.

The bearing stiffness is an important performance of the air bearings. Nakao[19] investigated the influence of the fluid pressure on the bearing stiffness. As air pressure changes, the bearing stiffness changes. If the air bearing has a positional offset, the stability of the spindle system will be affected, which in turn will affect the surface quality of the machined workpiece. Chen[20] revealed the oil pressure fluctuations of the hydrostatic slide producing waviness errors. In actual practice of the hydrostatic spindle, the bearings and the journals may not be properly aligned as a result of improper assembly, manufacturing errors and so on.
As a consequence, the rotor axis and the bearing axis are not in the same position. During the rotor rotating, the spindle system will generate tilting motions, which should be investigated[21]. Feng[22] presented the tilting motion of water-lubricated bearings, and investigated the effects of tilting ratio, rotary speed, and eccentricity ratio on the static and dynamic performances of the bearings. In ABS, a motor rotor and an air bearing are coupled as a body, which shows the multi-field and multi-parameter coupling relationship[23].

The stability of the air supply pressure is important role in the ultra-precision machining process, which directly affects the processing performance. The air supply equipment are mainly composed of air compressor, freezing dryer, filter and so on. The detailed forms are shown in Fig. 2. In the air supply system, the device for storing air uses an air storage tank, and the tank is a volume body that cushioning, storing and stabilizing the compressed air, which can avoid the frequent loading and unloading of the air compressor. Although the air storage tank is set as a stable air pressure volume, air flow is difficult to output smoothly, and there is a certain degree of air pressure fluctuation in the output. In addition, as air outputs, air pressure in the air tank will decrease, the pressure of the air tank will change, and the output air flow will also change. As a result, air supply pressure to the machine tool will decrease. Because the air supply pressure decreases, the bearing stiffness of the spindle system will be reduced and the spindle will be tilted as the bearing stiffness decreases. When air pressure in the air tank is lower than the set value of air pressure, the air compressor will start to work. As the air supply pressure increases, the stiffness of the spindle system increases and the air bearing returns to its original position.

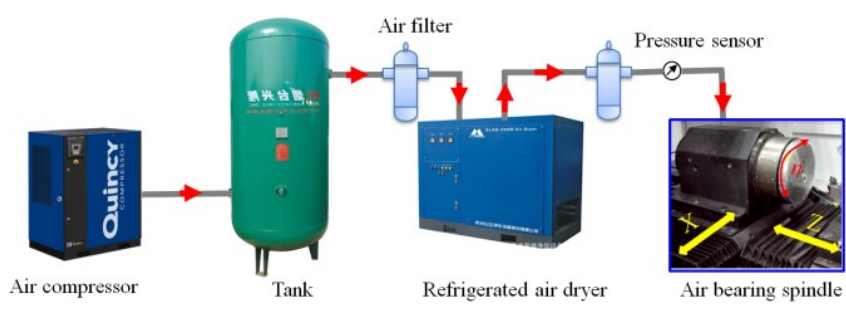

Fig.2 Schematic diagram of air supply for air bearings

Machining surface topography reflects directly the vibrations of the ultra-precision spindle during machining process, so ultraprecision machining is set as a method used to verify the dynamic performance of the ultra-precision spindle[24,25]. In machining processes, machining temperature has a significant influence on tool performance and the quality of a machining part as a result of the softening effect and diffusion, and measuring or predicting machining temperatures is important for the optimization of the machining process, Ning[26] presented an original analytical model for fast prediction of machining temperatures at two deformation zones in orthogonal cutting, namely the primary shear zone and the tool-chip interface, and investigated the machining temperatures of ultra-fine-grained titanium. Also, machining temperatures were predicted under various process conditions in orthogonal cutting 
tests[27,28]. The effects of machining temperature on the machining quality have been studied. This article will study the impacts of the spindle dynamic performance on the turning surface quality, considering the effects of MRE and APF.

PMSM and air supply system are the main components of air bearing spindles (ABS), so their performance affects the stability of ABS directly. This paper proposes a theoretical and experimental method to reveal the influence of the motor rotor eccentricity (MRE) and the air pressure variation (APF) as a whole on the machining workpiece surface. An ABS is used as a research object, which adopts a (10 poles and 12 slots) PMSM as the driving source. The characteristics of RMF and APF are presented and its effects on the air bearing are discussed. Besides, the mathematical model of the turning workpiece surface topography is established. The models take into account the effects of MRE and influence of APF, the mathematical model is used to predict the surface topography of the turning end face, and the forming profile reasons for the workpiece surface are described. In order to further verify the correctness of the theoretical analysis, this paper experimentally validates the influence of MRE and APF on the machining surface topography through turning experiments.

\section{RMF Induced by MRE}

\subsection{Analysis of motor MRE}

Because of the manufacturing tolerances, the rotor axis is not usually concentric with the stator axis, so the stator and the rotor axes are not parallel, resulting in the variation of flux path and equivalent air-gap length, and there will be a RMF between the rotor and the stator. An approach for calculating RMF is to derive the components of the magnetic force from the magnetic field. In the air gap of PMSM, the radial and tangential delta components of force densities can be evaluated using Maxwell stress method. As the magnets in the rotor have a much higher permeability than air, the tangential delta component of air-gap flux density is much smaller than the radial component, so the tangential delta flux density can be negligible[29,30].

In PMSM, the radial magnetic traction and the tangential magnetic traction acting on the motor rotor can be calculated according to Maxwell stress tensor method. The magnetic force caused by the varying air-gap electromagnetic fields can be decomposed into a delta tangential force and an RMF $F$. In common, the delta tangential force is smaller than RMF, thus it is neglected here. In order to clearly describe the motor rotor and the air gap, the sizes and positions of the rotor and stator are plotted with exaggeration, which is shown as Fig. 3. In this figure, points $O$ and $o$ represent the centers of the stator and the rotor, respectively; $e$ denotes the eccentricity between points $O$ and $o, o-x y$ represents the coordinate system of the rotor, which uses the center $o$ as the origin, $O-X Y$ represents the coordinate system of the stator, and $n$ represents the rotating speed of the spindle.

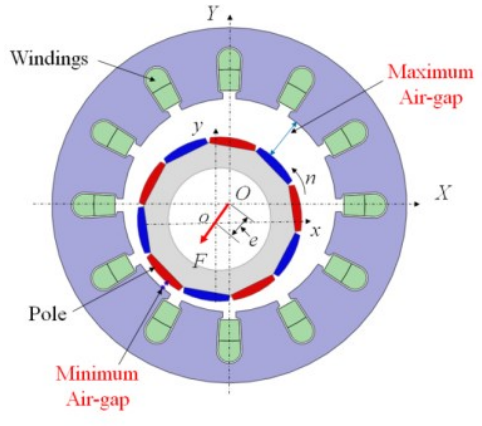

Fig. 3 Cross section of motor with MRE

\subsection{RMF calculation}

The electromagnetic force acting on the rotor is unevenly distributed, and it is not easy to calculate the value of RMF using a single analytical method. Based on the electromagnetic theory of the motor, Finite Element Method (FEM) is used to calculate RMF, because FEM can provide an accurate calculation for the dynamic motor responses when a motor is subjected to RMF, and a software named Ansoft Maxwell specifically is used to emulate motor characteristics, which considers the impacts of the saturation factor and current waveform, and accurately simulates the motor model[31,32]. A simplified motor model with a 10poles/12slots is carried out, and the permeability between stator and rotor is assumed to be infinite. Table 1[33] lists the detailed parameters of PMSM, and Ansoft Maxwell is used to simulate the motor model. Firstly, the rotor inner circle and the stator outer circle are selected as the boundary, which has no magnetic flux leakage. No rotor eccentricity and rotor eccentricity ( $e=80 \mu \mathrm{m}$, the air-gap length is set to $1 \mathrm{~mm}$ ) of the motor models are chosen to simulate the motor models. Motor models are simulated under load conditions, which operates at the same rated speed of $1000 \mathrm{rpm}$. At load conditions, the motor windings have a rated current of $6 \mathrm{~A}$ and 3 phases, and the simulations of the electromagnetic density cloud diagram is shown in Fig. 4 under the non-eccentric and eccentric cases.

Table 1 Parameters of PMSM

\begin{tabular}{ccc}
\hline Parameters & Rotor & Stator \\
\hline Inner diameter $(\mathrm{mm})$ & 50 & 66 \\
Outer diameter $(\mathrm{mm})$ & 64 & 110 \\
Poles/ slots & & $10 / 12$ \\
Total length $(\mathrm{mm})$ & \multicolumn{2}{c}{} \\
Air-gap[mm] & 1000 \\
Rated angular velocity $(\mathrm{r} / \mathrm{min})$ & 1 & \\
Rated power[kW] & \multicolumn{3}{c}{} \\
Winding form & 3-phase of double layer windings \\
\hline
\end{tabular}
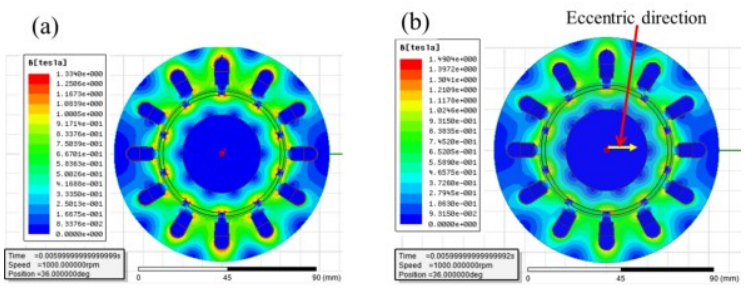

Fig. 4 Electromagnetic cloud (a) No eccentricity (b) Eccentricity ( $e=80 \mu \mathrm{m})$ 
Fig. 5 describes RMF with no eccentricity, which represents RMF with load condition. It can be seen from that RMF values are very small, almost all less than $4 \mathrm{~N}$. However, in the case of MRE, the RMF values become large. As shown in Fig. 6, the RMF has ripples of 10 peaks in one cycle, and the average values become greater than $90 \mathrm{~N}$.

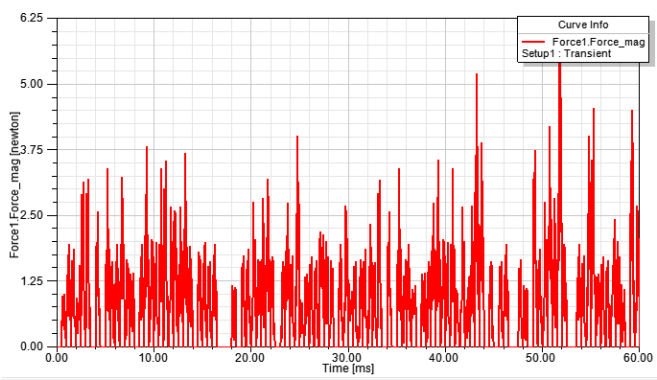

Fig. 5 RMF curve of motor rotor (no MRE)

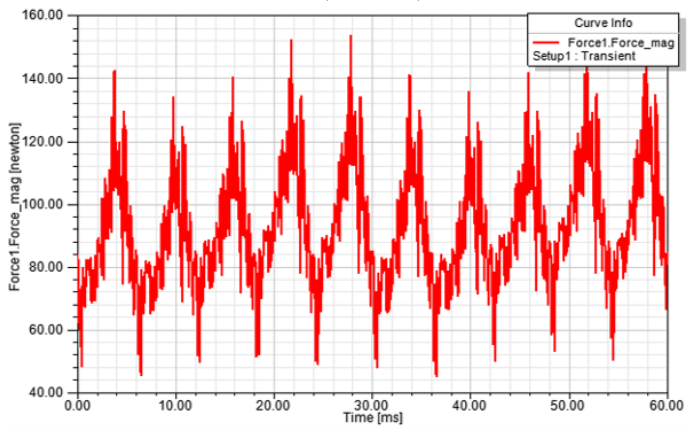

Fig. 6 RMF curve of motor rotor (MRE, $e=80 \mu \mathrm{m})$

\section{RMF effects on ABS in $\mathrm{Z}$ axis direction}

\subsection{Spindle tilt under RMF}

In order to further illustrate the influence of RMF on the machining performance, the machining principle diagram of ABS under the action of RMF is described in Fig.7. As shown in Fig.7, ABS with no eccentricity is a solid model, and the workpiece axis and the motor rotor axis is in the coincidence position. However, due to presence of RMF or center of gravity offset, the spindle is tilted, so the center axis of the workpiece and the motor rotor change the position. The spindle system has tilt as described in blue model, shown in Fig. 7. In the figure, the blue lines of the spindle indicate that $\mathrm{ABS}$ is subjected to the deformation caused by RMF, and the solid line structure indicates the initial position of the ABS. As Fig. 6 shows the simulation results of RMF $F$, it can be seen that the spindle system will periodically swing around its centroid $M$.

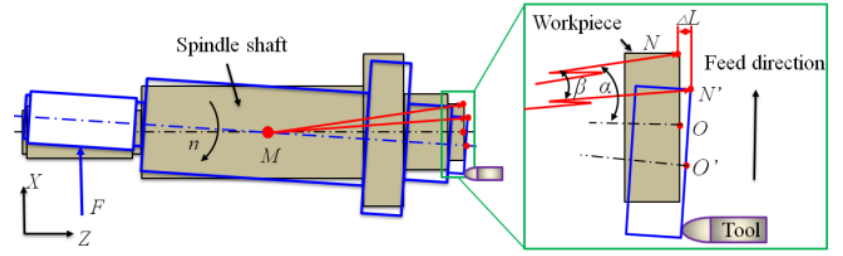

Fig. 7 Spindle shaft tilt caused by RMF

\subsection{Simulation of workpiece surface topography}

To discuss effects of RMF on the workpiece surface quality, a mathematical model of the surface topography is established and simulated. In the ideal case, the tool path on the workpiece surface is an Archimedes spiral, but the tool path on the workpiece surface will change due to effects of RMF, which reflects on the workpiece surface for the $Z$-direction offset vibrations. In order to simulate the surface topography, the rotational speed of ABS is set to $n$, the turning time is set to $t$, the feed rate of the feed system is set to $f$, the vibration frequency of spindle axial drift is set to $\omega$ (related to the fluctuation frequency of RMF), the amplitude of the vibration is set to $\Delta L$, and the inclination slope of ABS is set to $k$. According to the above parameters, the mathematical formula of the surface topography can be obtained by Eq. (1). According to Eq. (1), the coefficient $k$ affects the profile of the machined surface topography. When coefficient $k$ is set less than 0 , the surface topography is simulated by MATLAB and the result is shown in Fig. 8, which has a concave in the workpiece center.

$$
\left\{\begin{array}{c}
x=(n \cdot f \cdot t / 60) \cdot \cos (2 \pi \cdot n \cdot t / 60) \\
y=(n \cdot f \cdot t / 60) \cdot \sin (2 \pi \cdot n \cdot t / 60) \\
z=\Delta L \cdot \sin (\omega \cdot \pi \cdot t)+k \cdot t
\end{array}\right.
$$

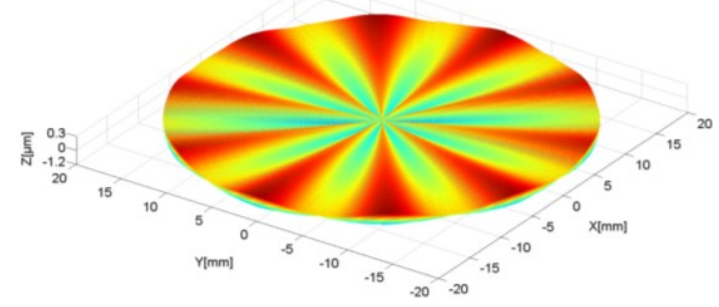

Fig. 8 Simulation of workpiece surface topography

\section{Prediction of surface topography considering RMF and APF}

\subsection{Air pressure variation}

To analysis air flow of the spindle system, a cross-sectional view of the air supply of ABS is established. The air bearing adopts the orifice throttling form, the specific structure is shown in Fig.9, and the partial enlarged view is expressed in Fig.9(A). The journal bearing adopts four rows of throttle holes to support spindle shaft in the axial direction, 12 throttling holes are evenly distributed in each row, and the diameter size is $D_{l}=\Phi 100 \mathrm{~mm}$. The thrust bearing adopts front and rear thrust bearings, each row evenly distributes 12 throttling holes, and the thrust bearing shaft diameter is $2 r_{l}=\Phi 200$ $\mathrm{mm}$. The compressed air forms air film lubrication when flowing through the orifice, and withstands the axial load. The throttle parameters are shown in Table 2[33]. In order to study the performance of the spindle system, the detail requirements for the mechanical structure are put forward: the axial stiffness is better than $300 \mathrm{~N} / \mu \mathrm{m}$, and the journal stiffness is better than $300 \mathrm{~N} / \mu \mathrm{m}$. 


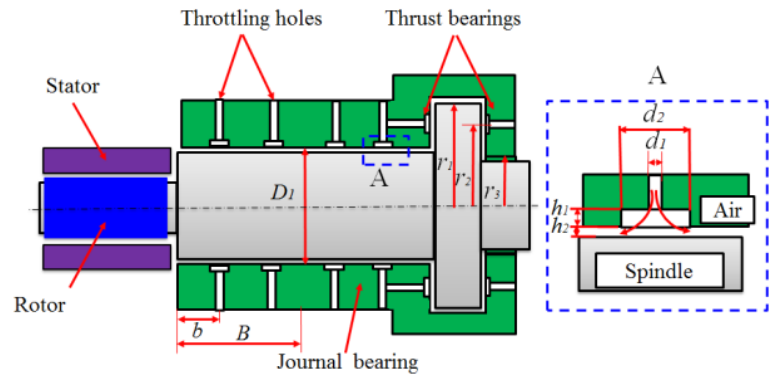

Fig. 9 Schematic illustrations of air bearings in ABS

Table 2 Main film parameters

\begin{tabular}{cccc}
\hline $\begin{array}{c}\text { Throttling groove } \\
\text { height } h_{l}(\mu \mathrm{m})\end{array}$ & $\begin{array}{c}\text { Film thickness } \\
h_{2}(\mu \mathrm{m})\end{array}$ & $\begin{array}{c}\text { Orifice Diameter } \\
d_{l}(\mathrm{~mm})\end{array}$ & $\begin{array}{c}\text { Throttling groove } \\
\text { diameter } d_{2}(\mathrm{~mm})\end{array}$ \\
\hline 50 & 15 & 0.2 & 8 \\
\hline
\end{tabular}

Air bearings use orifice throttling structures, and the structure of the journal bearing is shown in Fig. 9. Multi-row holes of air supply are used, the number of air holes per row is set to $Z=12$, the ratio of radial bearing length to diameter $B / D_{l}=1$, and $B=D_{l}=100$ $\mathrm{mm}$. The diameter of the orifice is $d_{l}=0.2 \mathrm{~mm}$, the throttling groove diameter is $d_{2}=8 \mathrm{~mm}$, air supply pressure is $P_{s}=0.6 \mathrm{Mpa}$, atmospheric pressure is $P_{a}=0.1 \mathrm{Mpa}$, the air gap of air bearings is $h_{2}=12 \mu \mathrm{m}$, the temperature is set to $T=288 \mathrm{~K}$, the air viscosity is set to $\mu=20^{*} 10^{-}$ ${ }^{6} \mathrm{~Pa} . \mathrm{s}$, the universal air constant is set to $\mathrm{R}=0.287 \mathrm{~kJ} /(\mathrm{kg} * \mathrm{~K})$, and $K g$ is set to 0.6. The flow rate calculation can be obtained by Eq. (2) [26], and air consumption of journal bearings is $0.015 \mathrm{~kg} / \mathrm{s}$.

$$
q_{m j}=\frac{\pi D_{1} h_{2}^{3}}{12 \mu R T l} K_{g}\left(P_{s}-1\right)\left[K_{g}\left(P_{s}-1\right)+2\right]
$$

The thrust bearings use symmetrical single-row air supply holes, and the specific structure is shown in Fig. 9. The number of single-row air holes is $Z=12$, the ratio of outer diameter to inner diameter is $R_{l}=r_{I} / r_{3}=2.0$, and the outer diameter is set to $2 r_{l}=\Phi 100$ $\mathrm{mm}$. The orifice diameter is set to $d_{l}=\Phi 0.2 \mathrm{~mm}$, the air supply pressure is $P_{s}=0.6 \mathrm{Mpa}$, the atmospheric pressure is $P_{a}=0.1 \mathrm{Mpa}$, the air gap is set to $h_{2}=12 \mu \mathrm{m}$, and the flow rate of the thrust bearings can be calculated by Eq. (3), and air consumption of thrust bearings is $0.011 \mathrm{~kg} / \mathrm{s}$. The spindle system and feed system of the ultraprecision machine tool studied in this paper use air bearings. After calculation of the total consumption, the air consumption is 0.052 $\mathrm{kg} / \mathrm{s}$, and the air consumption of the two feed systems is $0.045 \mathrm{~kg} / \mathrm{s}$.

$$
q_{m t}=\frac{\pi h_{0}^{3}}{3 \mu R \operatorname{Tn}\left(r_{2} / r_{1}\right)} K_{g}\left(P_{s}-1\right)\left[K_{g}\left(P_{s}-1\right)+2\right]
$$

The general pneumatic system can be divided into three parts: air supply system, air storage system, and air exhaust system. The air supply part mainly uses the air compressor to feed compressed air to the air tank, which is the power source of the pneumatic system. The air storage part is composed of an air tank, a filter drying device, etc. The main role is to store a certain pressure air, eliminate the air pulse from the air compressor, cool the compressed air and collect the condensed water. Exhausting air flow of air tank can be calculated using the following formula as
Eq. (4).

$$
V=Q_{1} \cdot T_{1} /\left(P_{M}-p_{S}\right)
$$

Where $V$ is the volume of the air tank (unit: Liter), $Q_{1}$ is the flow rate in the deflation phase $(\mathrm{L} / \mathrm{min}) ; P_{M}$ is the maximum preset pressure in the air tank when the compressor stop working (bar), $P_{2}$ is the lowest pressure value for the spindle working (bar); $T_{l}$ is the time for maintaining the normal operation of the pneumatic system after stopping the air supply.

When the air pressure in the air tank decreases to $P_{s}$, the air compressor starts to inflate the air tank. At the same time, the air tank also exhausts. When the air pressure in the tank reaches the set value, the air compressor will stop working. The time of air filling the air tank is calculated as Eq. (5).

$$
Q_{2} \cdot T_{2}+p_{s} \cdot V-Q_{1} \cdot T_{2}=V
$$

Where $Q_{2}$ is the air flow rate $(\mathrm{L} / \mathrm{min})$ to the air tank, and $T_{2}$ is air charge time of the compressor.

Therefore, the working cycle time $T$ of the air compressor is equal to the sum of the inlet time and the exhaust time of the air tank, which is shown in Eq. (6).

$$
T=T_{1}-T_{2}
$$

The spindle shaft uses air bearings, and the air tank pushes compressed air through the orifice to float the air bearings. When the air pressure in the tank decreases to a predetermined value, the compressor will work. When the pressure in the tank reaches a predetermined value, the compressor will stop working. The air pressure of the air storage tank firstly increases, and then the compressor stops working. As air exhausts, the air pressure in the air tank will slowly decrease. When the air pressure decreases to the given value, the compressor will begin to work. The air tank is inflated, and the air tank pressure gradually rises.

\subsection{Analysis of bearing capacity of thrust air bearings}

The thrust bearing of ABS adopts the small throttling orifice, and the single-side thrust bearing uniformly distributes 12 orifices. The load bearing capacity changing under different air supply pressures are analyzed[28]. The bearing capacity under different air pressure is calculated for the film thicknesses. As the inlet air pressure increases, the bearing capacity increases gradually and almost linearly changes. As the supply pressure increases, the air flow also increases. Due to the axial asymmetry of the spindle system itself, when the supply pressure changes, the bearing capacity changes, which causes the spatial position state of the spindle system to change, resulting in a tilt, which exhibits a periodic zigzag loop on the machining workpiece surface.

\subsection{Simulated surface topography}

Because of MRE in PMSM, the deviation of gravity center of the spindle system and the change of the air pressure supply, it can be seen from the simulation results that the three factors coupled together make the surface topography model more complicated. If $t$ 
is in the range $\left[N^{*} T, N^{*} T+T_{1}\right]$, the mathematical model for the surface topography is expressed as Eq. (7). If $t$ is in the range $\left[N^{*} T\right.$ $\left.+T_{1},(N+1) * T\right]$, the mathematical model for the surface topography is expressed as Eq. (8).

$$
\begin{gathered}
\left\{\begin{array}{c}
x=(n \cdot f \cdot t / 60) \cdot \cos (2 \pi \cdot n \cdot t / 60) \\
y=(n \cdot f \cdot t / 60) \cdot \sin (2 \pi \cdot n \cdot t / 60) \\
z=\Delta L \cdot \sin (\omega \pi \cdot n \cdot t)+k \cdot t-k_{1}(t+N \cdot T)+b_{1}
\end{array}\right. \\
\left\{\begin{array}{c}
x=(n \cdot f \cdot t / 60) \cdot \cos (2 \pi \cdot n \cdot t / 60) \\
y=(n \cdot f \cdot t / 60) \cdot \sin (2 \pi \cdot n \cdot t / 60) \\
z=\Delta L \cdot \sin (\omega \pi \cdot n \cdot t)+k \cdot t+k_{2}(t+N \cdot T)+b_{2}
\end{array}\right.
\end{gathered}
$$

Where $N$ is set an integer greater than or equal to zero, $k_{l}$ is the slope when the bearing stiffness decreases, $k_{2}$ is the slope during the bearing stiffness rise, $b_{1}$ and $b_{2}$ are the initial values of the simulation, respectively.

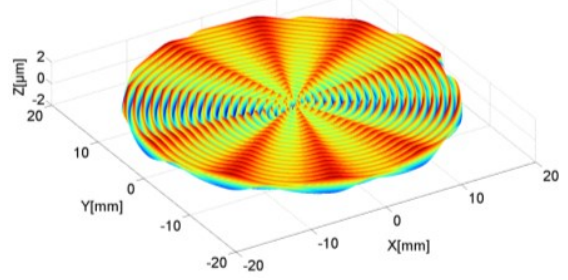

Fig. 10 Simulation of the surface topography considering air pressure variation

The workpiece surface quality is simulated according to Eqs. (7-8), and the simulation of the surface topography considering air pressure variation is shown in Fig. 10. Due to MRE, a periodical pattern of cyclical fluctuations is generated in the topography. Due to APF, the surface topography generates a cyclical change in the radial direction. In addition, due to the weight of the spindle system, the surface topography exhibits a groove shape as a whole.

\section{Experimental setup}

In order to further assess the performance of MRE and APF on ABS, a homemade ultra-precision ABS is developed, which is fixed on a feed system of an ultra-precision turning machine tool, which is used to obtain the ultra-precision optical components, and the performance parameters of the machine tool are shown in Table 3. The ultra-precision spindle is selected to study the influence of RMF and APF on machining workpiece surface. Machining is one of the most widely used manufacturing processes because of its fast speed and applicability to a broad class of materials. The machining processes of ultra-precision machine tool are shown in Fig. 11.

Table 3 Specifications of homemade machine tool

\begin{tabular}{ll}
\hline Travel & X-axis: $220 \mathrm{~mm} ;$ Z-axis: $300 \mathrm{~mm}$ \\
Straightness error & X-axis: $\leq 0.1 \mu \mathrm{m} / 100 \mathrm{~mm} ;$ \\
& Z-axis: $\leq 0.1 \mu \mathrm{m} / 100 \mathrm{~mm}$ \\
Spindle rotation accuracy & Axial: $\leq 0.08 \mu \mathrm{m} ;$ Radial: $\leq 0.08 \mu \mathrm{m}$ \\
\hline
\end{tabular}

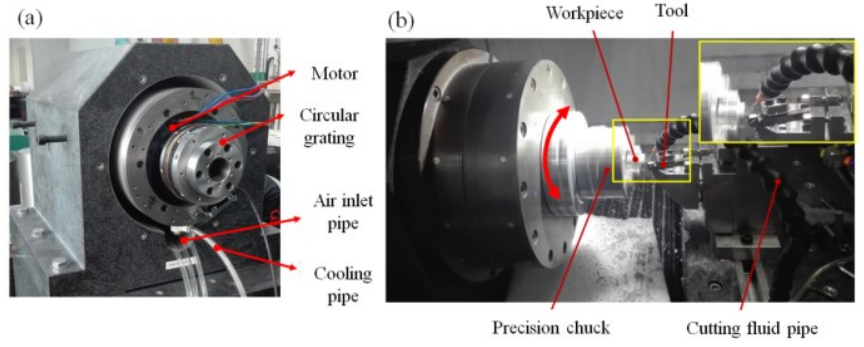

Fig. 11 Ultra-precision machining process:

The air pump station is used as air supply for air bearings. The spindle system uses a freeze dryer with a model of SLAD-4.5NF and a working pressure of $0.6 \mathrm{Mpa}-1.0 \mathrm{Mpa}$ (Hangzhou Shanli Purity Equipment Corpotation, China), and a fixed screw compressor with a model of QGF-10bar. The performance parameters are as follows: the air flow rate is $1.95 \mathrm{~m}^{3} / \mathrm{min}$, and the rated pressure is $1.0 \mathrm{Mpa}$. The maximum allowable working pressure of the air tank is $1.05 \mathrm{Mpa}$, the volume is $1 \mathrm{~m}^{3}$ (Yantai Xinglong Pressure Vessel Manufacturing Co. Ltd., China). The air supply to air-floatation system is set to $0.6 \mathrm{Mpa}$. Besides, the negative pressure principle is used to spray the machining fluid onto the workpiece during the machining process. ABS adopts a 10poles/12slots PMSM as the driving power, and an air bearing system is equipped with the spindle system. Monocrystal Diamond (MCD) is selected as the turning tool material, the radius of the circular tool is $3 \mathrm{~mm}$, the edge radius of the tool is less than $0.3 \mu \mathrm{m}$, and rake angle is set to $0^{\circ}$ and clearance angle is set to $8^{\circ}$. The workpiece material is Aluminum 7075, and all machined workpieces are tested using Phase Cam 6000 (4Sight $^{\mathrm{TM}}$ interferometer, 4D Technology Corporation, USA).

\section{Results and discussion}

\section{1 Experimental results}

Firstly, the rotation speed of ABS is set to $n=800 \mathrm{r} / \mathrm{min}$, the turning depth is set to $a p=6 \mu \mathrm{m}$, and the feed rate of feed system is set to $v_{f}=16.66 \mu \mathrm{m} / \mathrm{s}$. The tested surface topographies of the machined workpiece are shown in Figs. 12-13, the detection range of the surface topography is $9 \mathrm{~mm} \times 9 \mathrm{~mm}$, and two vertical lines passing through center of the surface topography are selected, which is shown in Fig.12a. The red curve is the lateral detection result, which is shown in Fig.12b. The detection result shows periodic ripples from the outside to the center of the workpiece, which can be seen in Fig.12a. Adjacent four cycles of annular ripples are selected to detect the distance, and the detection distance is $X=2.4 \mathrm{~mm}$. Besides, it can be seen from Fig. $12 \mathrm{~b}$ show that the resulting topography exhibits a concave shape from the outside to the inside center of the workpiece surface. The blue curve is the longitudinal detection result, which is shown in Fig. 12c. It can be seen that the longitudinal detection result and the horizontal test result show the same change. 


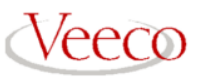

(a)
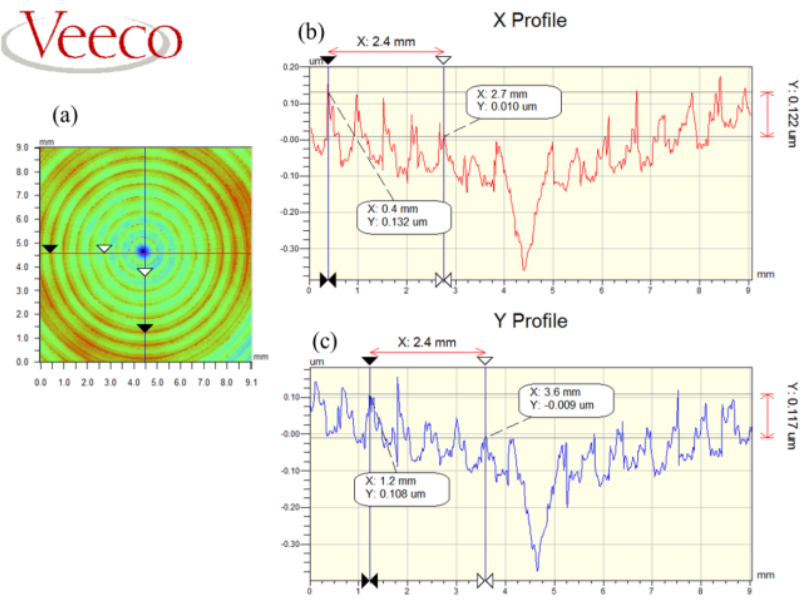

Fig. 12 Vertical line segments on machined surface topography $(\mathrm{n}=800 \mathrm{r} / \mathrm{min}$, $\mathrm{f}=16.66 \mu \mathrm{m}$ ):(a) selected two vertical lines, (b) lateral detection result,(c)longitudinal detection result.

Then, from the detection results of the workpiece surface, the workpiece surface center is used as the coordinate origin, a ring with a radius of $R=4.4 \mathrm{~mm}$ is extracted, and the circumferential surface topography of the detected workpiece is extracted and the result is shown in the red graph, which is shown in Fig.13b, and there are 10 uniformly distributed periodic ripples in a circle. At the same time, a line is drawn from the extension to the center on the surface topography, and the detected line segment is shown in Fig. 13c. The lateral distance between the two crests is selected and the detection distance is $X=0.564 \mathrm{~mm}$, which is the distance between adjacent two cycles of annular ripples in the surface topography. It can be seen that the detected value from edge to center of the workpiece surface is almost linearly reduced, the slope of the line segment is negative, and a concave is shown in the workpiece center.
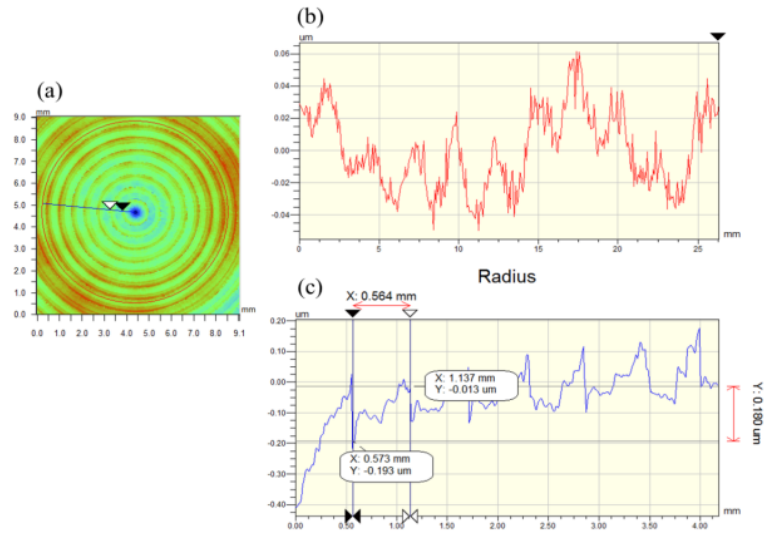

Fig. 13 A circle and a line on the machined surface topography $(n=800 r / m i n, f$

$=16.66 \mu \mathrm{m})$ :(a) selected vertical line, (b)circumferential surface topography,(c)detected line segment topography.
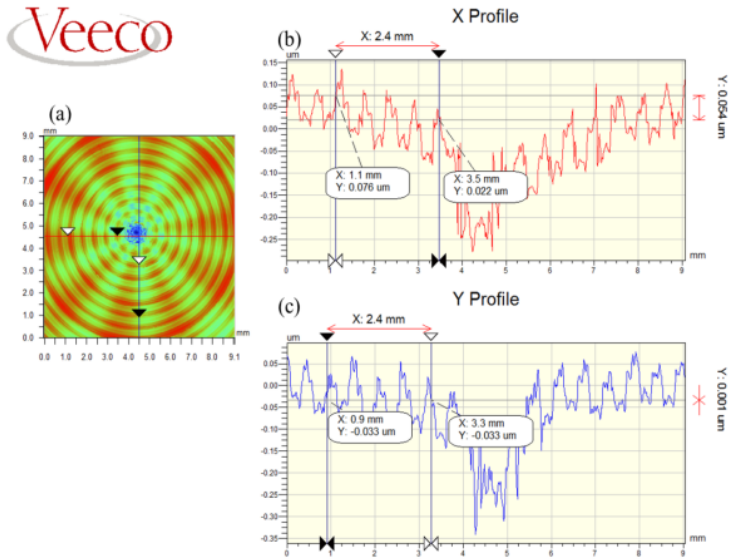

Fig. 14 Test result of a circle on the machined surface $(n=900 r / m i n, f=16.66 \mu m):(a)$ selected two vertical lines, (b) lateral detection result,(c)longitudinal detection result.
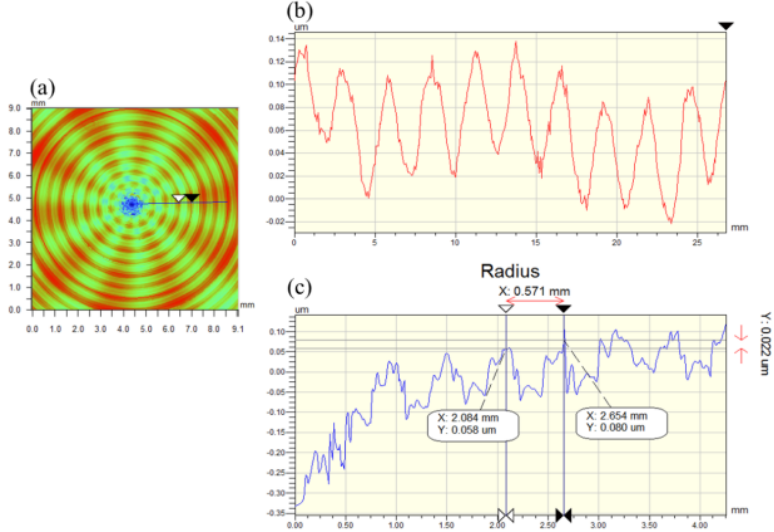

Fig. 15 Test result of a circle on the machined surface $(n=900 \mathrm{r} / \mathrm{min}, \mathrm{f}=16.66 \mu \mathrm{m})$ :(a) selected vertical line, (b)circumferential surface topography,(c)detected line segment topography.

The turning speed is set to $n=900 \mathrm{r} / \mathrm{min}$, and other turning parameters remain unchanged. The tested surface topographies are shown in Figs. 14-15. Two vertical lines passing through the center of the surface topography are selected, which is shown in Fig. 14a. The red curve is the lateral detection result, which is shown in Fig. 14b. The detection result shows periodic ripples from the outside to the workpiece surface center in Fig. 14a. In addition, it can be seen from the results show that the resulting topography exhibits a concave shape from the outside to the inside of the workpiece center. The blue curve is the longitudinal detection result shown in Fig. 14c. It can be seen that the longitudinal detection result and the horizontal test result show the same change. Also, the distance of adjacent four cycles of annular ripples is $X=2.4 \mathrm{~mm}$. 


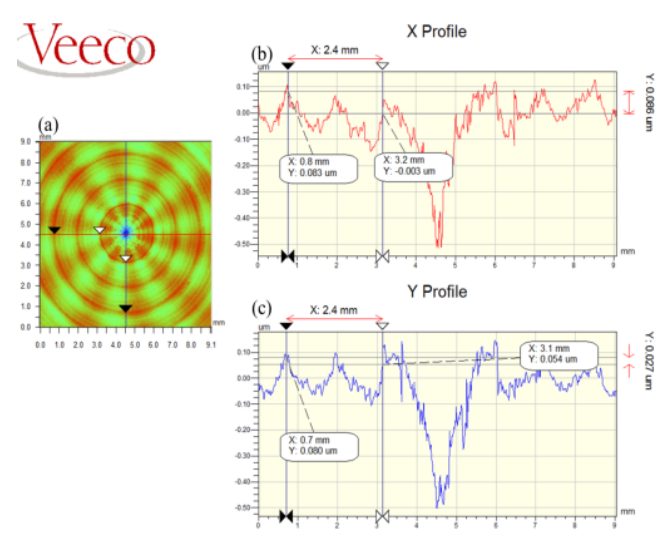

Fig. 16 Test result of a circle on the machined surface $(n=800 \mathrm{r} / \mathrm{min}, \mathrm{f}=33.33 \mu \mathrm{m})$ :(a) selected two vertical lines, (b) lateral detection result,(c)longitudinal detection result.

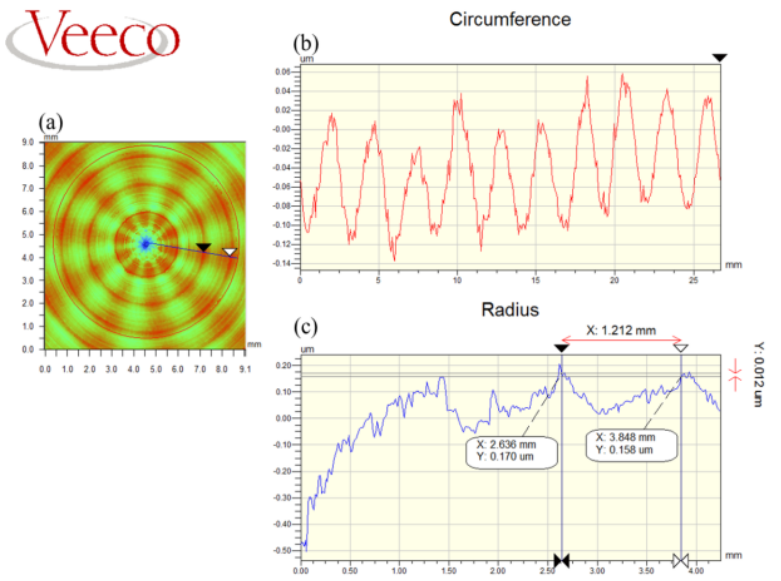

Fig. 17 Test result of a circle on the machined surface $(n=800 r / m i n, f=33.33 \mu m)$ :(a) selected vertical line, (b)circumferential surface topography,(c)detected line segment topography.

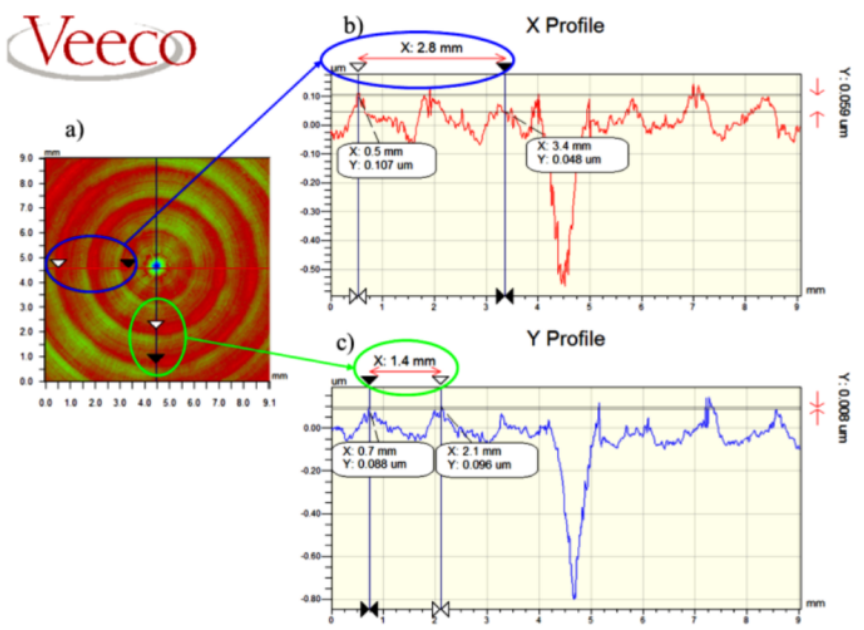

Fig. 18 Topography of a circle and a line on machined surface $(n=800 \mathrm{r} / \mathrm{min}$,

$v f=33.33 \mu \mathrm{m} / \mathrm{s}$, stop air supply of cutting fluid) :(a) selected two vertical lines, (b) lateral detection result,(c)longitudinal detection result.

The same detection method is the same as in Fig. 13, the center of the workpiece is used as the center of the coordinates, a ring with a radius of $R=4.4 \mathrm{~mm}$ is extracted in Fig. $15 \mathrm{a}$, and the circumferential surface topography of the detected workpiece surface is extracted and the result is shown in the red graph. As shown in Fig.15b, there are also 10 uniformly distributed periodic ripples in a circle. At the same time, the profile of the lateral curve changes as shown in the blue curve Fig.15c. The lateral distance between the adjacent annular ripples in the surface topography is selected and the distance result is $X=0.571 \mathrm{~mm}$.

The turning speed is set to $n=800 \mathrm{r} / \mathrm{min}$, the turning depth is set to $6 \mu \mathrm{m}$, the feed rate is set to $33.33 \mu \mathrm{m} / \mathrm{s}$, and other turning parameters remain unchanged. The tested surface topographies are shown in Figs. 16-17. Two vertical lines passing through the surface topography are selected, which is shown in Fig. 16a. The red curve is the lateral detection result shown in Fig. 16b. The detection result shows periodic ripples from the outside to the workpiece surface center shown in Fig.16a. In addition, it can be seen from the results show that the resulting topography exhibits a concave shape from the outside to the inside of the workpiece center. The blue curve is the longitudinal detection result, as shown in Fig. 16c. It can be seen that the longitudinal detection result and the horizontal test result show the same change. The distance of adjacent two cycles of annular ripples is $X=2.4 \mathrm{~mm}$.

The workpiece surface center is also used as the center of the coordinates, a ring with a radius of $\mathrm{R}=4.4 \mathrm{~mm}$ is extracted in Fig. $17 \mathrm{a}$, the circumferential surface topography of the detected workpiece is extracted and the result is shown in the red graph in Fig. 17b, and there are also 10 uniformly distributed periodic ripples in a circle. At the same time, the profile of the lateral curve changes as shown in Fig.17c. The lateral distance between the two adjacent crests is selected and the distance is $X=1.212 \mathrm{~mm}$.

To further explain the influence of the air pressure fluctuation on the workpiece surface shape, the air supply of the cutting fluid is stopped, and then the end face of the workpiece surface is turned. The turning spindle speed is $n=800 \mathrm{r} / \mathrm{min}$, the feed speed of the linear feed system is set to $v=33.33 \mu \mathrm{m} / \mathrm{s}$, and the cutting result is shown in Fig. 18. It can be seen from the tested results when the cutting fluid supply line is closed, the distance of the adjacent rings of the workpiece surface topography increases under the same turning parameters, increasing from the space $1.2 \mathrm{~mm}$ in Fig. 17 to $1.4 \mathrm{~mm}$ in Fig. 18. The reason is when stop the cutting fluid supply, it reduces the air pressure discharge, and the discharge cycle time of compressed air increases, so that the workpiece surface shows an increase distance of adjacent rings.

\subsection{Discussion}

Comparing the above machining inspection results, it can be seen that when the turning speed is set to $n=800 \mathrm{r} / \mathrm{min}$ and the feed speed is set to $v_{f}=16.66 \mu \mathrm{m} / \mathrm{s}$, the circumference of the workpiece surface exhibits periodic fluctuations, and there are 10 periodic ripples in a circle. In addition, the surface topography presents a periodic annular corrugation from the outside to the center of the workpiece surface, and the distance between the rings is $0.564 \mathrm{~mm}$. In addition, the workpiece surface presents a groove shape from the outside to the center of the workpiece surface. After changing the 
rotation speed, when the rotation speed is set to $n=900 \mathrm{r} / \mathrm{min}$, other turning parameters have no change. The surface topography is shown in Fig.16, and the tested result is similar as Fig.12. It can be seen that the spindle rotation speed has little effect on the workpiece surface topography. When the turning speed is set to $\mathrm{n}=800 \mathrm{r} / \mathrm{min}$, and the turning speed is set to $33.33 \mu \mathrm{m} / \mathrm{s}$, the test results are shown in Fig.16. Ten periodic ripples also exist in a circle of the workpiece surface, and the surface topography appears periodic ripple from the outside to the center of the workpiece workpiece surface. In the ring-shaped corrugations, the distance between adjacent ring-shaped corrugations is increased to 1.212 $\mathrm{mm}$. Due to the uneven amount of air consumed during processes, the zigzag ripple spacing on the surface topography is slightly different. Therefore, it can be seen when only the feed rate changes, and the distance between adjacent corrugated corrugations will be going to double.

There are periodic fluctuations in the workpiece surface, just as the theoretical analysis of the influence of MRE and the change of the inlet air pressure on the surface topography. Due to errors in design, manufacturing process and installation, the spindle system generates eccentric weight, and the axial position of the spindle deviates from the design position, causing the spindle system tilt along the mass center. In addition, due to eccentric installation of the motor, the motor stator acts on the motor rotor to generate RMF, and the force causes the motor rotor to produce periodic fluctuations. In addition, the air supply pressure of the air bearing spindle system is periodically changed. If air pressure in the air tank reaches the maximum allowable pressure, the air compressor will stop working. When the pressure value in the air tank drops to a predetermined value during air exhausting, the air compressor starts again. Although the air storage tank has the function of stabilizing the air pressure, the problem of pressure change is unavoidable. Therefore, the axial stiffness of the air bearing exhibits a periodic change, and the periodic circular ring corrugation changes appear on the workpiece surface during machining processes.

In addition, ultra-precision turning processing technology is also an important factor affecting processing quality. Among them, heat generation and tool grinding wear are also issues that need future research during the machining process. Therefore, the spindle heat generation, machining technology and tool grinding and grinding heat affect ultra-precision machining quality, which will be studied in-depth research in the future work.

\section{Conclusions}

The air bearing spindle (ABS) is a complex electromechanical system, which is the core system of ultra precision machine tools. Some research have been reported to study dynamic performance of $\mathrm{ABS}$, but lack of research on motor characteristics and air supply pressure, especially experimental research. MRE and APF have a very serious effect on the turning profile in the ultra-precision machining process. In this paper, a method of theoretical analysis and experimental verification is developed to study the effects of motor rotor eccentricity (MRE) and air pressure variation (APF) on the performance of the air bearing spindle (ABS). The following conclusions can be drawn:

1) A theoretical and experimental method for considering effects of MRE and APF on the machining workpiece surface topography has been proposed.

2) Due to MRE, the radial magnetic force causes the spindle shaft to tilt, causing periodic vibration during the rotation of the spindle.

3) The mathematical model of the workpiece surface topography under effects of MRE and APF has been established and simulation, also the effects of MRE and APF on workpiece surface topography have been verified by a prototype machine.

4) Due to effects of MRE, 10 periodic star patterns are generated on the turning surface, and ring patterns are generated by APF. Under different turning speeds, the periodic ripples appearing on the surface topography are different, and the formed change of the ring patterns is more obvious.

Acknowledgment The authors gratefully acknowledge financial support of the International Science \& Technology Cooperation Program of China (No. 2015DFA70630), the National Natural Science Foundation of China (Grant Nos.51505107 and 51705462), and Zhejiang Provincial Natural Science Foundation of China (Grant No. LQ20E050021).

\section{References}

1. Uriarte, L., Herrero, A., Zatarain, M., Santiso, G., Lacalle, L.N., Lamikiz, A., \& Albizuri, J. (2007). Error budget and stiffness chain assessment in a micromilling machine equipped with tools less than $0.3 \mathrm{~mm}$ in diameter. Precision Engineering, 31(1), 1-12.

2. Zhang, S. J., \& To, S. (2016). Spindle vibration influencing form error in ultraprecision diamond machining. Proceedings of the Institution of Mechanical Engineers Part C-Journal of Mechanical Engineering Science, 1989-1996, 203-210.

3. Kolar, P., Sulitka, M., \& Janota, M. (2011). Simulation of dynamic properties of a spindle and tool system coupled with a machine tool frame. International Journal of Advanced Manufacturing Technology, 54(1), 11-20.

4. Sung, S. J., Jang, G. H., Jang, J. W., \& Lee, H.L. (2013). Vibration and Noise in a HDD Spindle Motor Arising from the Axial UMF Ripple. IEEE Transactions on Magnetics, 49(6), 2489-2494.

5. Kim, D. J., Kim, H. J., Hong, J. P., \& Park, C.J. (2014). Estimation of Acoustic Noise and Vibration in an Induction Machine Considering Rotor Eccentricity. IEEE Transactions on Magnetics, 50(50), 857-860.

6. Xu, X. B., Liu, J.H., \& Chen, S. (2019). Internal model control for reduction of bias and harmonic currents in hybrid magnetic bearing. Mechanical Systems and Signal Processing, 115:70-81.

7. Fu, L., Zuo S. G., Deng, W. Z., \& Wu, S.L. (2016). Modeling and Analysis of Electromagnetic Force, Vibration, and Noise in Permanent-Magnet Synchronous Motor Considering Current Harmonics. IEEE Transactions on Industrial Electronics, 63(12),7455-7466.

8. Mystkowski, A., Kierdelewicz, A., Jastrzebski, R. P., Dragasius, E., \& Eidukynas, D. (2018). Flux measurement and conditioning system for 
heteropolar active magnetic bearing using Kapton-foil Hall sensors. Mechanical Systems and Signal Processing, 115, 394-404.

9. Kim, J. Y., Sung, S. J., \& Jang, G. H. (2012). Characterization and Experimental Verification of the Axial Unbalanced Magnetic Force in Brushless DC Motors. IEEE Transactions on Magnetics, 48(11): 3001-3004.

10. Li, Y., Lu, Q., Zhu, Z. Q., Wu, L.J., Li, G. J., Wu, D. (2015). Analytical Synthesis of Air-Gap Field Distribution in Permanent Magnet Machines With Rotor Eccentricity by Superposition Method. IEEE Transactions on Magnetics, 51(11): 1-4.

11. Li, J., \& Cho, Y. (2010). Dynamic reduction of unbalanced magnetic force and vibration in switched reluctance motor by the parallel paths in windings. Mathematics and Computers in Simulation, 81(2): 407-419.

12. Wu, Q.H., Sun, Y.Z, Chen, W.Q., Chen, G.D., Bai, Q.S., \& Zhang, Q.C. (2018). Effect of motor rotor eccentricity on aerostatic spindle vibration in machining processes. Proceedings of the Institution of Mechanical Engineers Part CJournal of Mechanical Engineering Science, 232(7), 13311342 .

13. Zhang, S. J., To, S., Cheung, C. F., \& Wang, H.T. (2012) Dynamic characteristics of an aerostatic bearing spindle and its influence on surface topography in ultra-precision diamond turning. International Journal of Machine Tools and Manufacture, 62(1): 1-12

14. Chen, W.Q., Liang, Y.C, Sun, Y.Z., Bai, Q.S., \& An, C. H. (2014). A novel dynamic modeling method for aerostatic spindle based on pressure distribution. Journal of Vibration and Control, 21(16), 3339-3347.

15. Liang, Y.C., Chen, W.Q., Bai, Q.S., Sun, Y. Z, Chen, G.D., Zhang, Q., \& Sun, Y. (2013). Design and dynamic optimization of an ultraprecision diamond flycutting machine tool for large KDP crystal machining. International Journal of Advanced Manufacturing Technology, 69(1-4): 237244.

16. Chen, G.D., Sun, Y.Z., Zhang, F.H., An, C. H., Chen, W.Q., \&Su, H. (2017). Influence of ultra-precision flycutting spindle error on surface frequency domain error formation. International Journal of Advanced Manufacturing Technology, 88(9), 3233-3241.

17. Chen, G.D, Sun, Y.Z., An, C.H., Zhang, F.H., Sun, Z.J., \& Chen, W. Q. (2016). Measurement and analysis for frequency domain error of ultraprecision spindle in a flycutting machine tool. Proceedings of the Institution of Mechanical Engineers Part B Journal of Engineering Manufacture, DOI: 10.1177/0954405416673102.

18. Xi, S.T., Cao, H.R., \&Chen, X.F. (2019). Dynamic modeling of spindle bearing system and vibration response investigation. Mechanical Systems and Signal Processing, 114, 486-511.

19. Nakao, Y., Suzuki, K., Yamada, K., \& Nagasaka, K. (2014). Feasibility Study on Design of Spindle Supported by High-Stiffness Water Hydrostatic Thrust Bearing. International Journal Automation Technology, 8, 530-538.

20. Chen, W.Q., Liang, Y.C., Sun, Y.Z., An, C.H., \& Chen, G.D. (2014). Investigation of the influence of constant pressure oil source fluctuations on ultra-precision machining. Proceedings of the Institution of Mechanical Engineers Part B Journal of Engineering Manufacture, 229(2):372-376.

21. Khanfir, H., Bonis, M., \& Revel, P. (2005). Improving waviness in ultra precision turning by optimizing the dynamic behavior of a spindle with magnetic bearings. International Journal of Machine Tools and
Manufacture,

$45(7), 841-848$

22. Feng, H. H., Xu, C. D., \&Wan, J. (2014). Mathematical Model and Analysis of the Water-Lubricated Hydrostatic Journal Bearings considering the Translational and Tilting Motions. Mathematical Problems in Engineering, 353769 .

23. Liu, J., \& Chen, X. (2014). Dynamic design for motorized spindles based on an integrated model. International Journal of Advanced Manufacturing Technology, 71(9), 1961-1974.

24. Zhang S., Li Z., Xiong Z. W., Suet T.(2019). A theoretical and experimental study of forced spindle vibration under unbalanced magnetic forces in ultraprecision machining. The International Journal of Advanced Manufacturing Technology, 103(9-12):4689-4694.

25. He C., Zong W.J.(2019). Influencing Factors and Theoretical Models for the Surface Topography in Diamond Turning Process: A Review. Micromachines, 10(5):288.

26. Ning J, Liang S.(2019). Predictive Modeling of Machining Temperatures with Force-Temperature Correlation Using Cutting Mechanics and Constitutive Relation. Materials, 12(2):284.

27. Ning J, Nguyen V, Huang Y, Hartwig K, Liang S.(2019). Constitutive modeling of ultra-fine-grained titanium flow stress for machining temperature prediction. Bio-Design and Manufacturing.2:153-160

28. Ning J., Nguyen V. , Liang S. Y. (2019). Analytical modeling of machining forces of ultra-fine-grained titanium. The International Journal of Advanced Manufacturing Technology, 101:627-636.

29. Valavi, M., Nysveen, A., Nilssen, R., Lorenz, R., \& Rølvåg, T. (2014). Influence of Pole and Slot Combinations on Magnetic Forces and Vibration in Low-Speed PM Wind Generators. IEEE Transactions on Magnetics, $50(5), 1-11$.

30. Chen, X., Yuan, S.H., \& Peng, Z.X. (2015). Nonlinear vibration for PMSM used in HEV considering mechanical and magnetic coupling effects. Nonlinear Dynam, 80(1-2) : 541-552.

31. Yunfei Wang. (1999). Gas lubricated theory and design manual of gas bearings. Beijing: Machinery Industry Press, 154-167.

32. Wu, Q. H., Sun, Y.Z., Chen, W.Q., Wang, Q., \& Chen, G.D. (2019). Theoretical prediction and experimental verification of the unbalanced magnetic force in air bearing motor spindles. Proceedings of the Institution of Mechanical Engineers Part B Journal of Engineering Manufacture, 233(3):2330 - 2344

33. Wu, Q. H., Sun, Y. Z., Chen, W.Q. Liu, H.T., \& Luo, X.C. (2019). An Mechatronics Coupling Design Approach for Aerostatic Bearing Spindles. International Journal Precision Engineering and Manufacturing, DOI: 10.1007/s12541-019-00098-w.

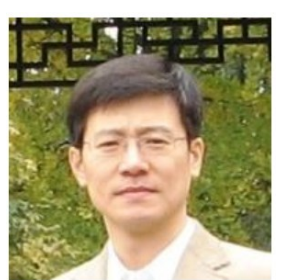

Yazhou Sun is currently a professor at School of Mechatronics Engineering, Harbin Institute of Technology, China. He received his $\mathrm{PhD}$ degree from Harbin Institute of Technology, China, in 2005. His research interests include ultraprecision machine tool and machining technology, and additive manufacturing. 


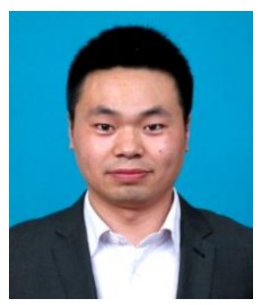

Quanhui Wu is currently a lecturer at School of Mechanical Engineering, Zhejiang University of Technology, China. He received his $\mathrm{PhD}$ degree from Harbin Institute of Technology, China, in 2019. His research interests include precision ultra-precision machining technology and electromechanical couplings.

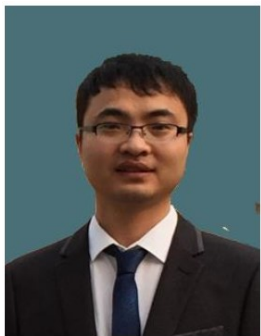

Wanqun Chen is currently an Associate Professor at School of Mechatronics Engineering, Harbin Institute of Technology, China. He received his $\mathrm{PhD}$ degree from Harbin Institute of Technology, China, in 2014. His research interests include ultra-precision machine design and dynamic analysis.

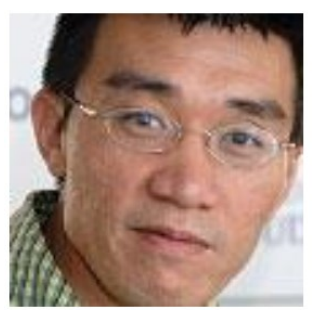

Xichun Luo is a Professor in ultra precision manufacturing at the University of Strathclyde (Glasgow), UK. He obtained his $\mathrm{PhD}$ at Leeds Metropolitan University (UK). His current research interests include design of high precision machines, ultra precision diamond turning and grinding of freeform optics, and nanomanufacturing research.

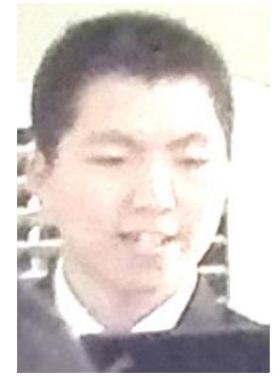

Guoda Chen is currently a lecturer at School of Mechanical Engineering, Zhejiang University of Technology, China. He received his $\mathrm{PhD}$ degree from Harbin Institute of Technology, China, in 2015. His research interests include ultra-precision machine dynamic analysis and ultra-precision machining technology. 